\title{
Kondo effect in multielectron quantum dots at high magnetic fields.
}

\author{
C. Tejedor ${ }^{1}$ and L. Martín-Moreno ${ }^{2}$ \\ ${ }^{1}$ Departamento de Física Teórica de la Materia Condensada, Universidad Autónoma de Madrid, 28049 Madrid, Spain. \\ ${ }^{2}$ Departamento de Física de la Materia Condensada, ICMA(CSIC), Universidad de Zaragoza, Zaragoza 50015, Spain.
}

\begin{abstract}
We present a general description of low temperature transport through a quantum dot with any number of electrons at filling factor $1<\nu<2$. We provide a general description of a novel Kondo effect which is turned on by application of an appropriate magnetic field. The spin-flip scattering of carriers by the quantum dot only involves two states of the scatterer which may have a large spin. This process is described by spin-flip Hubbard operators, which change the angular momentum, leading to a Kondo Hamiltonian. We obtain antiferromagnetic exchange couplings depending on tunneling amplitudes and correlation effects. Since Kondo temperature has an exponential dependence on exchange couplings, quantitative variations of the parameters in different regimes have important experimental consequences. In particular, we discuss the chess board aspect of the experimental conductance when represented in a grey scale as a function of both the magnetic field and the gate potential affecting the quantum dot.
\end{abstract}

PACS numbers: 72.15.Qm

\section{INTRODUCTION}

Kondo effect has been recently observed in quantum dots (QD) coupled to leads by anatying the temperature dependence of the conductance $\mathrm{B}$. $\mathrm{B}$. Kondo physics has been mainly detected for an odd number of electrons $N$ in the QD while for even $N$, the temperature dependence of the conductance is usually that corresponding to a normal system. This is interpreted in terms of the formation of singlets by pairs of electrons so that only when $N$ is odd a single electron remains unpaired. This last electron, in a state doubly degenerate by spin, is responsible for the Kondo behavior proposed_long time agot 10. However, some recent experiments 113 suggests a more complicated situation because Kondo effect is also observed for even $N$ in some situations. A particularly interesting experimental feature is the appearance, for a given $N$, of alternating high and low conductance valleys as a function of an external magnetic field $B 11$. 13. When $N$ is varied in \pm 1 , the high and low conductance valleys are interchanged. Therefore, the representation of the conductance (in a grey scale) as a function of both $B$ and a gate potential wich allows to vary $N$, takes the aspect of a chess bagrd1 13 .

Three models 1416 have been already proposed for understanding how Kondo effect might be possible for states of two electrons. In these approaches, the authors consider the situation of double degeneracy that a magnetic field can create between the singlet $\left(S_{z}=0\right)$ and the triplet $\left(S_{z}=1\right)$ states of the electronic pair. However, as shown below, the actual situation is, in general, rather more complicated due to correlation and tunneling amplitude effects.

In this paper we present a general description of the Kondo effect in a QD in the presence of a high magnetic field such that the filling factor is $1<\nu<2$. This is a regime in which experiments clearly show Kondo effect 11 . The description is valid for any number of electrons (even or odd without restriction to 1 or 2) and any value of the QD spin $S_{z}$ (not restricted to 0,1 for even $N$ or $1 / 2$ for odd $N$ ). Instead of describing spin-flip scattering in terms of spin-ladder operators $S^{( \pm)}$, we find a set of spin-flip Hubbard operators describing a collective spin effect of all the $N$ electrons contained in the QD. Despite both $N$ and $S_{z}$ can be very large, the scattering of carriers only produces transitions between two manybody states of the QD with $S_{z}$ differing in \pm 1 . These many-body states are well characterized theoretically in the regime $1<\nu<2$, and we find that the spin-flip process can be described by a Kondo Hamiltonian with antiferromagnetic couplings which depend on both tunneling amplitudes and correlation effects. As a consequence, the system presents a Kondo behavior with a Kondo temperature $T_{K}$ which we analyze in various limiting cases.

In section II we discuss the ground state (GS) of an isolated QD in the regime $1<\nu<2$. in Section III an effective Kondo Hamiltonian is obtained, by means of a scattering description of tunneling through the QD. Section IV contains a discussion on the exchange couplings and the Kondo temperature in the regime $1<\nu<2$. Section $\mathrm{V}$ is devoted to the explanation of the chess board aspect of the experimental conductance. A summary is given in section VI.

\section{QD SPECTRUM}

We consider $N$ electrons in the presence of a magnetic field and confined in a QD coupled to leads. The Hamiltonian is

$$
H=H_{Q D}+H_{L}+H_{T U N} .
$$

Within a lowest Landau level approach, an isolated parabolic QD with magnetic field and interaction between the electrons is described by (hereafter we take $\hbar=1)$ 


$$
\begin{aligned}
H_{Q D} & =\frac{N \Omega}{2}+\frac{\left(\Omega-\omega_{c}\right) M}{2}+g \mu_{B} B S_{z}+ \\
& \frac{1}{2} \sum_{m_{i}, \sigma_{i}} V_{m_{1} m_{2} m_{3} m_{4}} d_{m_{1}, \sigma_{1}}^{\dagger} d_{m_{2}, \sigma_{2}}^{\dagger} d_{m_{3}, \sigma_{3}} d_{m_{4}, \sigma_{4}} .
\end{aligned}
$$

The first two terms describe a single particle contribution depending on both the QD confinement $\omega_{0}$ and cyclotron $\omega_{c}$ frequencies through $\Omega=\sqrt{\omega_{c}^{2}+4 \omega_{0}^{2}}$. $\quad M$ is the third component of the total angular momentum. The third term is the Zeeman energy, depending on the Landé $g$-factor. In the last term, describing electronelectron repulsion, $d_{m, \sigma}^{\dagger}$ creates an electron with angular momentum $m$ and spin $\sigma$ in the QD. The Coulomb interaction matrix elements $V_{m_{1} m_{2} m_{3} m_{4}}$ have a typical energy scale $e^{2} / \varepsilon l_{B}$, where $\varepsilon$ is the dielectric constant and $l_{B}=1 / \sqrt{m \Omega}$ the magnetic length. These interactions decrease with the increasing width $w$ of the quantum well in which the QD has been built up.

$H_{L}=\sum_{k, \sigma} \varepsilon_{k, \sigma} c_{k, \sigma}^{\dagger} c_{k, \sigma}$ describes, in a single-particle approach, the leads having electrons with quantum numbers $k, \sigma$ occupying states up to the Fermi energy $\varepsilon_{F}$.

A crucial role is going to be played by the tunneling part of the Hamiltonian

$$
H_{T U N}=\sum_{k, m, \sigma} V_{m}\left(d_{m, \sigma}^{\dagger} c_{k, \sigma}+c_{k, \sigma}^{\dagger} d_{m, \sigma}\right)
$$

in which we neglect any dependence on $k$ of the tunneling amplitudes $V_{m}$ (taken as real positive) but we retain the dependence on $m$ because is going to produce physical consequences.

$H_{Q D}$ can be numerically diagonalized for a significantly broad range of $N$, as discussed in many theoretical papers 17 . An example (similar results are obtained for an odd number of electrons) is shown in Fig. 1 and 2, for $N=8$, which is the regime in which some experiments 11 have been performed, although some others 13 involve a larger number of electrons. In order to have access to all the possibilities for the GS, it is convenient to discuss a case with strong interaction effects, so that we present results for a QD with $w=0$. The case of an arbitrary finite width $w$ can be qualitatively discussed from these results: correlated GS tend to disappear when $w$ increases. Fig. 1 shows the phase diagram of the possible GS between $\nu=1$ and $\nu=2$. The wider regions of the upper part of the phase diagram correspond to compact states which are favored by large Zeeman coupling

$$
\left|C_{N-K}^{K}\right\rangle=\prod_{m=0}^{K-1} d_{m, \downarrow}^{\dagger} \prod_{m=0}^{N-K-1} d_{m, \uparrow}^{\dagger}|0\rangle
$$

where $|0\rangle$ is the vacuum state. In going from left to right, one finds successively the states $\left|C_{4}^{4}\right\rangle(\nu=2),\left|C_{5}^{3}\right\rangle,\left|C_{6}^{2}\right\rangle$, $\left|C_{7}^{1}\right\rangle$ and $\left|C_{8}^{0}\right\rangle(\nu=1)$. Strong interaction effects are manifested in the appearance, lower in the phase diagram, of the narrower regions corresponding to different skyrmion-like states 18.19

$$
\left|S K_{N, K, \pm}^{P}\right\rangle=\mathcal{N}_{P, \pm}\left(\Lambda_{ \pm 1}^{\dagger}\right)^{P}\left|C_{N-K}^{K}\right\rangle
$$

of topological charge 1. $\mathcal{N}_{P, \pm}$ is a normalization constant and

$$
\Lambda_{ \pm 1}^{\dagger}=\sum_{m}(m+1)^{\mp 1 / 2} d_{m+1, \downarrow}^{\dagger} d_{m, \uparrow}
$$

Adjacent regions correspond to values of $P$ which differ in one unit and, as a consequence, their spins also differ in 1. Dashed lines in Fig. 1 depict, for two different values of $\omega_{0}$, the evolution of the GS for a GaAs QD within the range of magnetic fields (perpendicular to the QD) given at the edges of the lines.

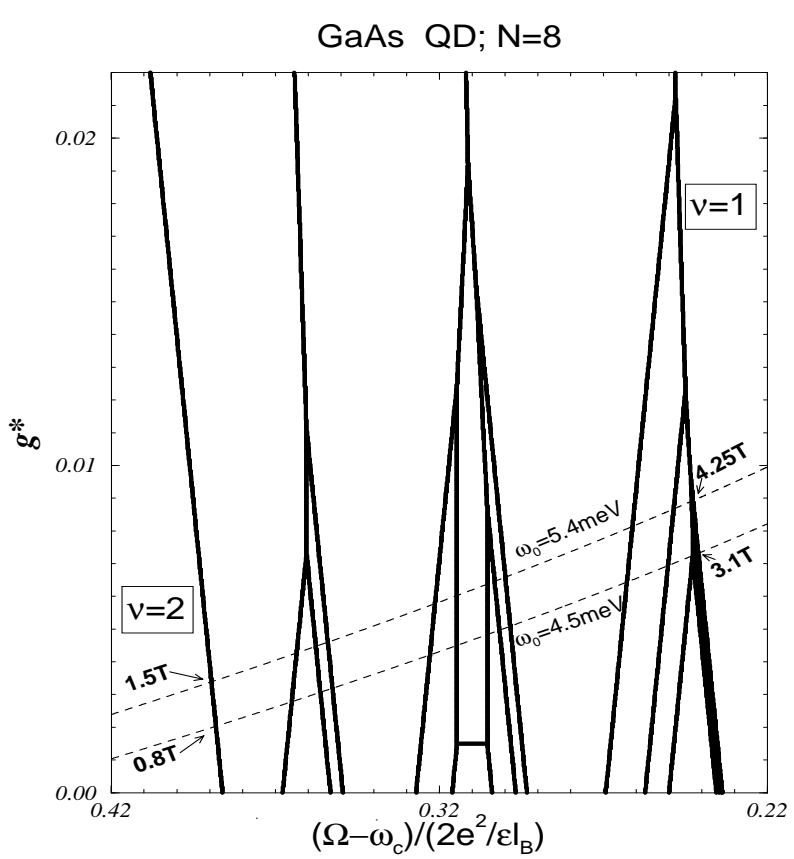

FIG. 1. Phase diagram of the possible GS of a GaAs QD with 8 electrons at $2>\nu>1$. The axes contain kinetic $\left(\Omega-\omega_{c}\right) /\left(2 e^{2} / \varepsilon l_{B}\right)$ and Zeeman $g^{*}=g \mu_{B} B /\left(e^{2} / \varepsilon l_{B}\right)$ contributions to the energy. The (upper) wider regions correspond to compact states from $\left|C_{4}^{4}\right\rangle(\nu=2)$ at the left to $\left|C_{8}^{0}\right\rangle$ $(\nu=1)$ at the right. The (lower) narrower regions correspond to different skyrmion-like states. Dashed lines depict, for two different values of the QD confinement frequency $\omega_{0}$, the evolution of the GS within the range of magnetic fields given at the edges of the lines.

Fig. 2 shows the evolution of the GS properties from $\nu=2$ to $\nu=1$ for the system of Fig. 1 with $\omega_{0}=5.4 \mathrm{meV} 20$. The GS energy $E_{G S}$ has a kink any time a crossing of states occurs. Since these kinks are not obvious in Fig.2(b), we represent, in part (a), the energy splitting $\Delta E=E_{e x c}-E_{G S}$ between the lowest excited state and the GS. The spin $S_{z}$ and the third component $M$ of the total angular momentum of the GS are also given in (c) and (d) respectively. 
GaAs QD; $\omega_{0}=5.4 \mathrm{meV} ; \mathrm{N}=8$
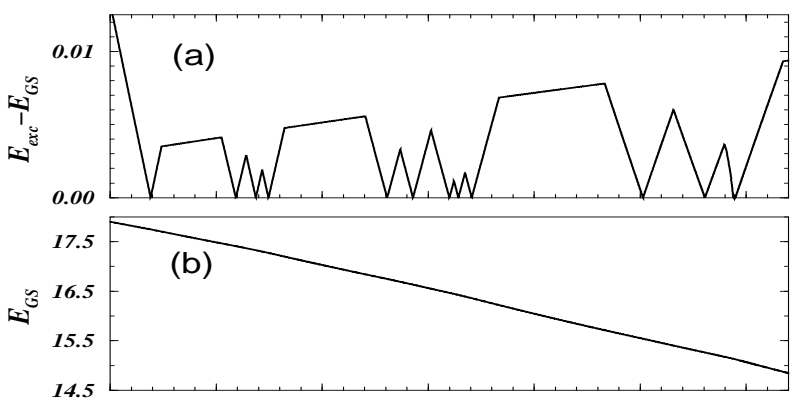

(c)
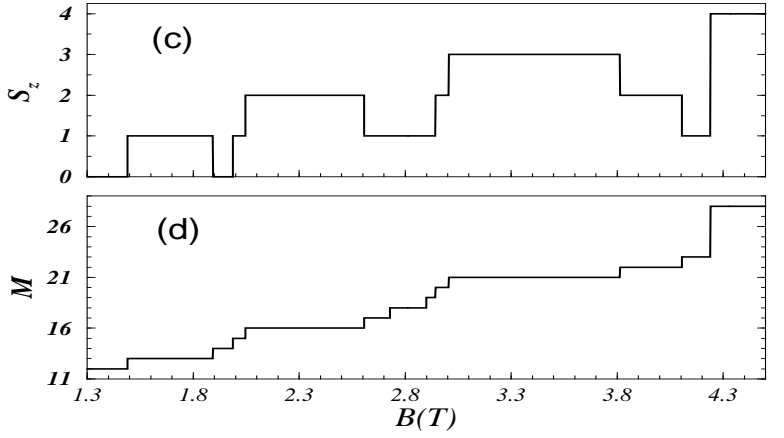

FIG. 2. Evolution with the magnetic field $B$ of different magnitudes for the GS of a GaAs QD with $N=8$ and $\omega_{0}=5.4 \mathrm{meV}$. Energies are measured in units of $e^{2} / \varepsilon l_{B}$. The left side of the figure corresponds to $\nu=2$ and the right side to $\nu=1$.

Independently of the particular details, there is a general trend for any $N$ : when the magnetic field increases from $\nu=2$ to $\nu=1$, the GS changes many times (at least $N / 2$ changes corresponding to electrons $N / 2$ flipping their spins one by one). In other words, for $2>\nu>1$ there are several crossings of the lowest energy states. This means that, for many values of the field, the GS is doubly degenerated (or non degenerated but having an excited state with extremely low excitation energy).
Moreover, in almost all the cases, these two states have spins differing in 1 while they have different $M$.

\section{KONDO HAMILTONIAN}

As a consequence of the above numerical result, the main physics of the problem is captured by a two level system approach 21. One considers the QD in the regime $2>\nu>1$ as having two degenerate (or almost degenerate) GS's $\left|G S_{\Uparrow}^{N}\right\rangle$ and $\left|G S_{\Downarrow}^{N}\right\rangle$ with $M_{\Uparrow} \neq M_{\Downarrow}$ and spins differing in 1, i.e. $\left\langle G S_{\Uparrow}^{N}\left|S_{z}\right| G S_{\Uparrow}^{N}\right\rangle=\left\langle G S_{\Downarrow}^{N}\left|S_{z}\right| G S_{\Downarrow}^{N}\right\rangle+1$. $H_{T U N}$ mixes these GS's with states $|N \pm 1\rangle$ in which $N \pm 1$ electrons are in the QD. In our two level system description, the tunneling Hamiltonian is projected on the subspaces subtended by the two GS's for $N$ electrons and the connecting $|N \pm 1\rangle$ electron states. In this process, we consider the connecting $|N \pm 1\rangle$ states as non-degenerate22. Using the notation $\Sigma \equiv\{\Uparrow, \Downarrow\}$, we introduce the tunneling spectral amplitudes

$$
\begin{aligned}
& \Delta_{-, \Sigma}=\sum_{m, \sigma} V_{m}\left\langle N-1\left|d_{m, \sigma}\right| G S_{\Sigma}^{N}\right\rangle \\
& \Delta_{+, \Sigma}=\sum_{m, \sigma} V_{m}\left\langle N+1\left|d_{m, \sigma}^{\dagger}\right| G S_{\Sigma}^{N}\right\rangle
\end{aligned}
$$

and the spin-flip Hubbard operators

$$
X_{\Sigma, \Sigma^{\prime}}=\left|G S_{\Sigma}^{N}\right\rangle\left\langle G S_{\Sigma^{\prime}}^{N}\right| .
$$

From projected tunneling Hamiltonian $\bar{H}_{T U N}$, the interaction between the QD with $N$ electrons and the leads is studied by a standard scattering description 23 . An effective coupling is obtained by summing up to all the possible intermediate states $|I\rangle$, in a second order perturbation approach:

$$
\begin{aligned}
& H_{e f f}=\sum_{I} \frac{\bar{H}_{T U N}|I\rangle\langle I| \bar{H}_{T U N}}{E_{G S}^{N}-E_{I}} \\
& =\sum_{k, k^{\prime}, \Sigma}\left[\frac{\Delta_{-, \Sigma}^{2} \delta_{k, k^{\prime}} X_{\Sigma, \Sigma}}{E_{G S}^{N}-E^{N-1}-\varepsilon_{F}}+\frac{\Delta_{+, \Sigma}^{2} c_{k^{\prime}, \sigma}^{\dagger} c_{k, \sigma}}{E_{G S}^{N}-E^{N+1}+\varepsilon_{F}}+\sum_{\Sigma^{\prime}}\left(\frac{\Delta_{+, \Sigma} \Delta_{+, \Sigma^{\prime}}}{E^{N+1}-\varepsilon_{F}-E_{G S}^{N}}+\frac{\Delta_{-, \Sigma} \Delta_{-, \Sigma^{\prime}}}{E^{N-1}+\varepsilon_{F}-E_{G S}^{N}}\right) X_{\Sigma, \Sigma^{\prime}} c_{k^{\prime}, \sigma^{\prime}}^{\dagger} c_{k, \sigma}\right]
\end{aligned}
$$

where, due to spin conservation, $\Sigma$ has the same direction than $\sigma$ and $\Sigma^{\prime}$ the same than $\sigma^{\prime}$. The first term is simply a constant. The second term (involving $c_{k^{\prime}, \sigma}^{\dagger} c_{k, \sigma}$ ) represents a potential scattering which does not involve any spin flip. These two terms are identical to the ones appearing when building up an $s d$ Hamiltonian from the Anderson Hamiltonian in the case of $N=123$. As it is usually done in that case, one can forget about these two terms which do not contain anything important for the physics we want to address.

The interesting physics is included in the third term of (9) which is a Kondo Hamiltonian

$$
H_{K}=\sum_{k, k^{\prime}}\left[J\left(X_{\Uparrow, \Downarrow} c_{k^{\prime} \downarrow}^{\dagger} c_{k, \uparrow}+X_{\Downarrow, \uparrow} c_{k^{\prime}, \uparrow}^{\dagger} c_{k, \downarrow}\right)+J_{\Uparrow} X_{\Uparrow, \uparrow} c_{k^{\prime}, \uparrow}^{\dagger} c_{k, \uparrow}+J_{\Downarrow} X_{\Downarrow, \Downarrow} c_{k^{\prime}, \downarrow}^{\dagger} c_{k, \downarrow}\right] .
$$

with exchange couplings

$$
J=\frac{\Delta_{+, \Uparrow} \Delta_{+, \Downarrow}}{E^{N+1}-\varepsilon_{F}-E_{G S}^{N}}+\frac{\Delta_{-, \Uparrow} \Delta_{-, \Downarrow}}{E^{N-1}+\varepsilon_{F}-E_{G S}^{N}} ; \quad J_{\Sigma}=\frac{\Delta_{+, \Sigma}^{2}}{E^{N+1}-\varepsilon_{F}-E_{G S}^{N}}+\frac{\Delta_{-, \Sigma}^{2}}{E^{N-1}+\varepsilon_{F}-E_{G S}^{N}} .
$$


$H_{K}$ is a spin-flip scattering Hamiltonian in which the GS of the scatterer flips its spin by means of $X_{\Uparrow, \Downarrow}$ and $X_{\Downarrow, \Uparrow}$ (as shown in Fig. 3) and, at the same time, changes its total angular momentum. Both the difference between $M_{\Uparrow}$ and $M_{\Downarrow}$, and the correlation effects included in the tunneling spectral amplitudes, are the reason why one must use spin-flip Hubbard operators instead of the usual spin-ladder operators $S^{( \pm)}$.

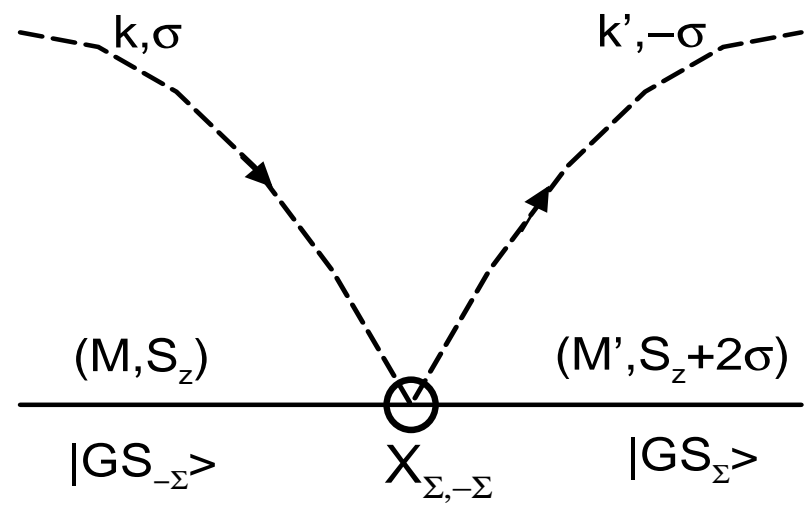

FIG. 3. Simplest diagram representing the spin-flip scattering described by the $X_{\Sigma,-\Sigma}=\left|G S_{\Sigma}^{N}\right\rangle\left\langle G S_{-\Sigma}^{N}\right|$ Hubbard operators. The base line depicts the evolution of the momentum and spin of the scatterer (the QD). Dashed lines depict carriers with spin $\sigma= \pm 1 / 2$ at the leads. Spin conservation requires that $\sigma$ has the same direction than $\Sigma$.

A crucial question is the sign of the exchange couplings (11). With our definitions (), all the tunneling spectral amplitudes are positive. Therefore, the signs of the exchange couplings are determined by the denominators. As, in the considered situation, the lowest energy corresponds to having $N$ electrons in the $\mathrm{QD}$, all the intermediate states $|I\rangle$, with $N \pm 1$ electrons in the QD and $\mp 1$ electron at the Fermi level of the leads, have higher energy. Therefore, we have a very important result: $H_{K}$ has positive effective exchange couplings. So, a Kondo Hamiltonian (10) with antiferromagnetic couplings (11) has been obtained. The conclusion is: for any values of both $N$ and spin, the $Q D$ in the regime $2>\nu>1$ presents Kondo physics.

\section{EXCHANGE COUPLINGS AND KONDO TEMPERATURE}

Since the Hamiltonian (10) is formally equivalent to the standard Kondo Hamiltonian for $N=1$, the temperature dependence of the conductance (as any other measurable property) presents now characteristics similar to the one found in those experiments 3 which have been interpreted in terms of only one electron. The important issue to be discussed is the characteristic energy scale, $T_{K}$, which is determined by the antiferromagnetic couplings.
$J$ and $J_{\Sigma}$ depend on both the energy difference $E_{G S}^{N}-$ $E^{N \pm 1} \mp \varepsilon_{F}$, and tunneling spectral amplitudes $\Delta_{-, \Sigma}$. The former is practically independent on both $N$ and $\Sigma$. Therefore, it does not imply any significant difference with respect to the well known case of $N=1$. However interesting physical differences appear due to $\Delta_{-, \Sigma}$. Let us analyze the different situations in which the QD can be found:

i) (Upper part of Fig. 1) When the Zeeman interaction is large (for instance having a large inplane component of the magnetic field), the GS's are always compact states

$$
\left|G S_{\Uparrow}^{N}\right\rangle=\left|C_{N-K}^{K}\right\rangle ;\left|G S_{\Downarrow}^{N}\right\rangle=\left|C_{N-K-1}^{K+1}\right\rangle .
$$

This is the simplest case in which

$$
X_{\Uparrow, \Downarrow}=(-1)^{K} d_{N-K-1, \uparrow}^{\dagger} d_{K, \downarrow}
$$

and similarly for the other Hubbard operators. We have also assumed, $|N-1\rangle=\left|C_{N-K-1}^{K}\right\rangle$ and $|N+1\rangle=\left|C_{N-K}^{K+1}\right\rangle$ (in other case the quantum numbers of the $d^{\dagger}$ operators must be changed accordingly) and the signs are taken according with definitions (8). Due to the lack of correlation effects in the GS's, the tunneling spectral amplitudes are

$$
\Delta_{-, \Uparrow}=\Delta_{+, \Downarrow}=V_{N-K-1} ; \Delta_{-, \Downarrow}=\Delta_{+, \Uparrow}=V_{K} .
$$

There is a difference between the antiferromagnetic couplings $J, J_{\Uparrow}$ and $J_{\Downarrow}$ due to the fact that $N-K-1>K$. It is easier to tunnel from the leads to spin up states $(m=N-K-1)$ within the QD because they are in the outer region of the QD while the first available spin down state $(m=K)$ is in the inner region of the QD. This tunneling amplitude effect js not described by previous models of Kondo at finite $\mathrm{B} 4 \mathbf{4}-16$, but has long been more broadly recognized both experimentally and theoretically for $\mathrm{QD}$ with $2>\nu>1$.

Although in this regime the QD does not present any correlation effects, the antiferromagnetic couplings are still a function of total energies and tunneling amplitudes. Since the Kondo temperature depends exponentially on $J$ 23, it is a very sensitive magnitude with respect to many parameters as $w, \omega_{0}, B, V_{m}$ etc. However, this is not different from the case of just one electron in the QD in which experiments 1 show $T_{K}$ to be in the range of $1 \mathrm{~K}$. In any case, one can predict variations in $T_{K}$ when the regime changes as discussed below.

ii) (Lower part of Fig. 1) When the Zeeman interaction is small enough, the fS's are skyrmion-like states of topological charge one 18.19 :

$$
\left|G S_{\Uparrow}^{N}\right\rangle=\left|S K_{N, K, \pm}^{P}\right\rangle ;\left|G S_{\Downarrow}^{N}\right\rangle=\left|S K_{N, K, \pm}^{P+1}\right\rangle
$$

The Hubbard operators $X_{\Sigma, \Sigma^{\prime}}$ have complicated, but analytical, expressions in terms of $\Lambda_{ \pm 1}^{\dagger}$.

When $N$ is very large, correlation effects provoke that $\Delta_{-, \Sigma}$ tends to zero (for instance $\Delta_{-, \Sigma} \propto[N \ln N]^{-1 / 2}$ for $P=0$ ) implying a quenching of the antiferromagnetic 
couplings due to the orthogonalization catastrophe. As a consequence, Kondo effect should not be observed when the number of electrons within the $\mathrm{QD}$ is large because the Kondo temperature is extremely small in this case.

Even more interesting is the effect for a reduced number of electrons, for instance $N=8$ as both in Fig. 11, 2 and in the experiment 11 . In this case, correlation effects do not destray Kondo effect but reduce $\Delta_{-, \Sigma}$ up to a factor of two 17 . The reduction of $J$ and $J_{\Sigma}$ implies that Kondo temperatures are significantly smaller than for the case $i$ ) of compact states. In practice, one can move from the skyrmions regime ii) (lower part of Fig 11) to the compact regime i) (upper part of Fig 11) by increasing an inplane component of the magnetic field. As a consequence of the above analysis, one should detect a clearly measurable increase of $T_{K}$ during this process.

iii) There is a rather unusual case, in which, for intermediate Zeeman interaction and only for a few values of the magnetic field, the degenerate GS's are one skyrmion and one compact state, i. e.:

$$
\left|G S_{\Uparrow}^{N}\right\rangle=\left|S K_{N, K,+}^{P}\right\rangle ;\left|G S_{\Downarrow}^{N}\right\rangle=\left|C_{N-K+1}^{K-1}\right\rangle .
$$

This happens, for instance, in the last step to the right in Fig. 2. In this case, the two GS have spins differing in more than 1. Therefore, they can not be obtained from the same $|N-1\rangle$ by creating one electron with spin either up or down. In other words, one of the two GS's has a tunneling spectral amplitude equal to zero so that, for tunneling effects there is only a non degenerate GS and Kondo effect does not occur.

A practical difference between the compact and skyrmion cases resides in the probability of finding two degenerate (or almost) GS's. As shown in Fig. 1, the crossing regions are much closer to each other for skyrmions (lower part of Fig. 1) than for compact states (upper Fig. 1). To work with a magnetic field in which Kondo effect exists is more probable in the skyrmion regime than in the compact one. However, once Kondo regime is found in the compact regime, the effect is more robust because its $T_{K}$ is higher than the one for skyrmions.

\section{CHESS BOARD BEHAVIOR OF THE CONDUCTANCE}

A very characteristic feature of some experiments 11,13 is an alternating high-low conductance sequence as a function of $B$ for a given temperature and number of electrons. When $N$ is varied in \pm 1 , the high and low conductance valleys are interchanged. Since a common way of representing the experimental results is to use a color-intensity scale for the conductance as a function of both $B$ and a gate potential which varies $N$, the data present a chess board aspect 11, 13. This occurs in a broad range of filling factors and number of electrons.
In order to understand such an experimental behavior, let us start by applying our description for the regime $1<\nu<2$. Since the chess board is observed 11,13 in samples with large $w$, skyrmion-like states are high energy excitations. GS's are restricted to be given just by one configuration, i. e. $\left[S^{(-)}\right]^{j}\left|C_{N-K}^{K}\right\rangle$ states, where $j$ is an integer (for practical purposes one can concentrate in the cases $j=0$ and 1$)$. At first sight, the consideration of both $\left|C_{N-K}^{K}\right\rangle$ and $S^{(-)}\left|C_{N-K}^{K}\right\rangle$ seems unnecessary because these states have an energy difference equal to the Zeeman coupling so that they are never degenerate. However, in a broad range of magnetic fields, these two states are precisely the two lowest energy states (see e.g. Fig. 2).

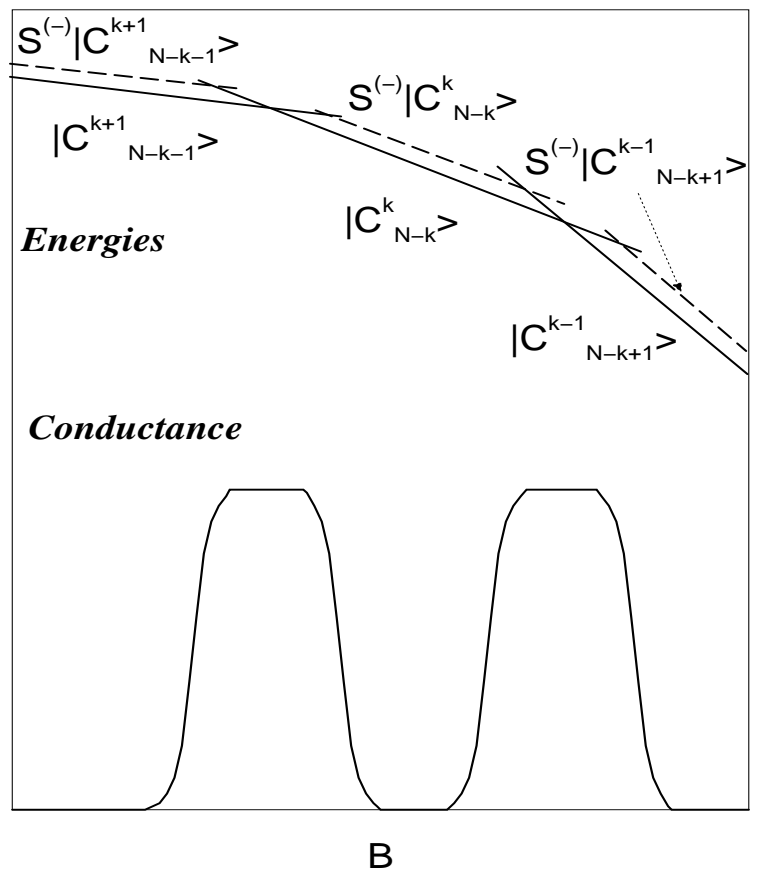

FIG. 4. Schematic evolution with the magnetic field of the two lowest energy states of a QD (upper part) and the conductance (lower part) in the range $T_{K}>T>\Delta E^{2} / T_{K}$ (see text).

In the two level system approach21 in which the two lowest states are separated by an energy splitting $\Delta E$, Kondo-like behavior appears only when the experimental temperature is between $(\Delta E)^{2} / T_{K}$ and $T_{K}$ (provided $\left.\Delta E<T_{K}\right)$ in order to have significant occupation of the two states. The quantitative application of the model would require the computation of both the energy splitting $\Delta E$ and $T_{K}$ which, as mentioned above, are very sensitive to many experimental parameters. Instead, a general understanding of the chess board is obtained from the following qualitative explanation depicted schematically in Fig. 4: when magnetic field is varied, the two lowest levels are in two alternating situations depending whether the system is around or far from a crossing of GS's. Around a crossing, the two lowest levels 
correspond to states $\left|C_{N-K-1}^{(K+1)}\right\rangle$ and $\left|C_{N-K}^{K}\right\rangle$ while far from the crossing they are $\left|C_{N-K}^{K}\right\rangle$ and $S^{(-)}\left|C_{N-K}^{K}\right\rangle$. In the former case, the two lowest levels can be connected by the tunneling Hamiltonian through low energy states $|N \pm 1\rangle$ with $\Delta_{ \pm, \Sigma}=V_{K} V_{N-K-1}$. In this case, the system presents high Kondo-like conductance. In the second case of being far from the crossings, the two lowest states are $\left|C_{N-K}^{K}\right\rangle$ and $S^{(-)}\left|C_{N-K}^{K}\right\rangle$. The coupling $J$ between the two states is very weak for two reasons: first, the connecting states $|N \pm 1\rangle$ are highly excited states implying very large denominators in $J$ and, second, since $S^{(-)}\left|C_{N-K}^{K}\right\rangle$ is a linear combination of different configurations, $\Delta_{ \pm, \Sigma}$ is significantly reduced by factors proportional to $1 / N$. These two effects produce, in this region, a very small $J$ and, consequently, an exponentially negligible $T_{K}$ so that the measured conductance is not Kondo-like but instead is quenched. This originates the alternating behavior experimentally observed for fix gate voltage (i. e. number of electrons in the QD) and varying magnetic field. The chess board aspect also implies alternating low-high conductance regions for fix $B$ and varying gate voltage, which is due to the fact that crossings for $N \pm 1$ electrons occur for magnetic fields roughly midway from crossings for $N$ electrons 17 .

Since Kondo-like behavior only occurs when the experimental temperature is in the range between $(\Delta E)^{2} / T_{K}$ and $T_{K}$, a clear prediction of our scheme is that the highly conducting regions of the chess board would become narrower for decreasing temperature due to the lower limit condition.

The sequence of alternating high-low conductance has been observed also at very low magnetic field $(B \simeq 0.5 \mathrm{~T})$ and large number $(N \simeq 50)$ of electrons 13 . So, a complete understanding of the experimental situation requires the extension of our framework to $\nu>2$. We are currently involved in this task which implies to take into account GS's more complicated than simple compact states in the lowest Landau level.

\section{SUMMARY}

We have presented a general description of the Kondo effect for any number of electrons in a QD at filling factor $1<\nu<2$. Collective spin effects of the $N$ electrons are described in terms of a set of spin-flip Hubbard operators which also change the third component of the total angular momentum. Our conclusion is that, for any number of electrons within the QD, the spin-flip scattering of carriers only produces transitions between two (either compact or skyrmion-like) states of the QD with spins differing in 1 . This process can be described by a Kondo Hamiltonian with exchange couplings that, we show, are antiferromagnetic and depend on both correlation and tunneling amplitude effects. As a consequence, the system presents a Kondo behavior with an experimentally accessible $T_{K}$. The increase of $T_{K}$ with an inplane com- ponent of the magnetic field should allow the detection of the transition from a skyrmion-like regime to a compact state regime. Finally, we present a qualitative explanation for the chess board aspect of the experimental conductance when represented in a grey scale as a function of both the magnetic field and the gate potential affecting the quantum dot.

\section{ACKNOWLEDGEMENTS}

We are grateful to J. Weis and L. P. Kouwenhoven for providing us with experimental information before its publication. This work was supported in part by MEC of Spain under contract No. PB96-0085, Fundacion Ramon Areces and CAM under contract No. 07N/0026/1998.

${ }^{1}$ D. Goldhaber-Gordon, J. Gores, M. A. Kastner, H. Shtrikman, D. Mahalu, U. Meirav, Nature 391, 156 (1998); Phys. Rev. Lett. 81, 5225 (1998).

${ }^{2}$ S. M. Cronenwett, T. H. Oosterkamp, and L. P. Kouwenhoven, Science 281, 540 (1998).

${ }^{3}$ F. Simmel, R. H. Blick, J. P. Kotthaus, W. Wegscheider and M. Bichler, Phys. Rev. Lett. 83, 804 (1999).

${ }^{4}$ L. I. Glazman and M. E. Raikh, Pis'ma Zh. Eksp. Teor. Fiz. 47, 378 (1988) [JETP Lett. 47, 452 (1988)].

${ }^{5}$ T. K. Ng and P. A. Lee, Phys. Rev. Lett. 61, 1768 (1988).

${ }^{6}$ A. Kawabata, J. Phys. Soc. Jpn., 60, 3222 (1991).

${ }^{7}$ S. Hershfield, J. H. Davies, and J. W. Wilkins, Phys. Rev. Lett. 67, 3720 (1991).

${ }^{8}$ Y. Meir, N. S. Wingreen, and P. A. Lee, Phys. Rev. Lett. 70, 2601 (1993).

${ }^{9}$ A. Levy-Yeyati, A. Martin-Rodero and F. Flores, Phys. Rev. Lett., 71, 2991 (1993).

${ }^{10}$ K. A. Matveev, Phys. Rev. B, 51, 1743 (1995).

${ }^{11}$ J. Schmid, J. Weis, K. Eberl, K. von Klitzing, Phys. Rev. Lett., 84, 5824 (2000).

12 S. Sasaki, S. De Franceschi, J. M. Elzerman, W. G. van der Wiel, M. Eto, S. Tarucha, and L. P. Kouwenhoven, Nature, 405, 764 (2000).

${ }^{13}$ W. G. van der Wiel, S. De Franceschi, T. Fujisawa, J. M. Elzerman, S. Tarucha, and L. P. Kouwenhoven, to be published.

${ }^{14}$ M. Pustilnik, Y. Avishai and K. Kikoin, Phys. Rev. Lett., 84, 1756 (2000).

${ }^{15}$ M. Eto and Y. V. Nazarov, Phys. Rev. Lett., 85, 1306 (2000).

${ }^{16}$ D. Giuliano and A. Tagliacozzo, Phys. Rev. Lett., 84, 4677 (2000).

${ }^{17}$ See for instance J.J.Palacios, L.Martin-Moreno, G.Chiappe and E.Louis and C.Tejedor, Phys. Rev. B, 50, 5760 (1994) and references therein.

18 J. H. Oaknin, L. Martin-Moreno and C. Tejedor, Phys. Rev. B, 54, 16850 (1996); erratum, 55, 15943 (1997). 
19 J. H. Oaknin, B. Paredes and C. Tejedor, Phys. Rev. B, 58, 13028 (1998).

${ }^{20}$ This is a typical value for QD as it was determined by single electron capacitance in R. C. Ashoori, H. L. Stormer, J. S. Weiner, L. N. Pfeiffer, S. J. Pearton, K. W. Baldwin and K. W. West, Phys. Rev. Lett., 68, 3088 (1992); ibid 71, 613 (1993).
${ }^{21}$ D. L. Cox and A. Zawadowski: Exotic Kondo effects in Metals (Taylor and Francis, London, 1999).

${ }^{22}$ Non-degeneracy of $|N \pm 1\rangle$ states is the most common case. If either $|N+1\rangle$ or $|N-1\rangle$ is also degenerate, the algebra is more complicated but the physics is the same.

${ }^{23}$ A. C. Hewson: The Kondo problem to Heavy Fermions (Cambridge University Press, Cambridge, 1993). 